\title{
Sustainable solutions for spa design, Dubai, United Arabic Emirates: building envelope optimization and impact energy evaluation
}

\author{
D. Tanzj, C. Clemente, F. Cumo \& G. Piras \\ Department C.I.T.E.R.A., Sapienza University of Rome, Italy
}

\begin{abstract}
In recent years, energy consumption for cooling buildings has become a real concern, particularly regarding the availability of electricity during high load hours. There is a growing sensitivity in architecture for approaches that respect the environment and pursue energy efficiency. Ventilation, like other passive cooling solutions, involves conceptualizing a building as a body able to both recognize and metabolize the resources of its environment. In this context, the role of technology is no longer a "value added" to architecture, but a tool integrated with it. For these reasons, my study and research efforts have been focused mainly on the development of sustainable technologies. The intention is to exploit the natural elements of a site: sun, soil and, particularly, air. Thus, the design of the building is done in steps, starting with the choice of exposure and then moving on to the shape, the study of shielding systems, and the observation of greenery outside. The goal of optimal wellness is to locate buildings in a manner that does not adversely affect the potential use of climatic resources and to position individual buildings so as to maximize the use of winds while maintaining consistent configuration of shape and geometry. Graphics were produced to conclude the study design and to demonstrate $\mathrm{CO}_{2}$ reduction.

Keywords: sustainable, archetypes, passive design, integrated, green energy, solar control, wellness, ventilation, energy efficiency, passive cooling, soil cooling, evaporative cooling, sustainable technologies, natural elements, climatic resource, insulating, induced ventilation, saving $\mathrm{CO}_{2}$.
\end{abstract}




\section{Introduction}

This proposal involves the designing of a wellness centre in Dubai using sustainable integrated solutions. The design theme was inspired by an interest in new forms of green energy, and attention was paid to typical Middle Eastern archetypes functions, such as solar control and ventilation. The project consists of the development of a resort designed as a set of housing units with common areas and a wellness spa. The area is located in one of the artificial islands of "The World" in front of Dubai City. Dubai is one of the seven United Arab Emirates (UAE). The homonymous capital is one of the fastest growing cities in the world. Its success is driven by its excellent geographical location, which makes it a connector to the main markets of Europe, Africa and Asia. Today, Dubai is a genuine work site, famous for an innovative spirit that enables architects and engineers to develop and implement projects while testing renewable technologies and new infrastructure solutions. Located about 16 meters above sea level, its territory is almost entirely desert. The appearance of reddish sand is due to a high concentration of iron oxide.

The climate in Dubai is extremely hot and humid, characterized by very low rainfall concentrated in the winter. The largest archipelago, "The World", is located about 4 kilometres from the coast of Dubai and is comprised of 310 artificial islands. Each island has an area of between 300,000 and 900,000 square feet. Each island constitutes a nation, and together they form the shape of the world map. The islands are independent, each with flexible interpretation regarding uses and urban forms. Nakheel [1] is the company engaged in the real estate development projects, and is responsible for a series of colossal achievements in Dubai. Great importance was placed on the analysis of the needs of various sectors in the territory and to the consequent development of forwardlooking projects based on emerged findings.

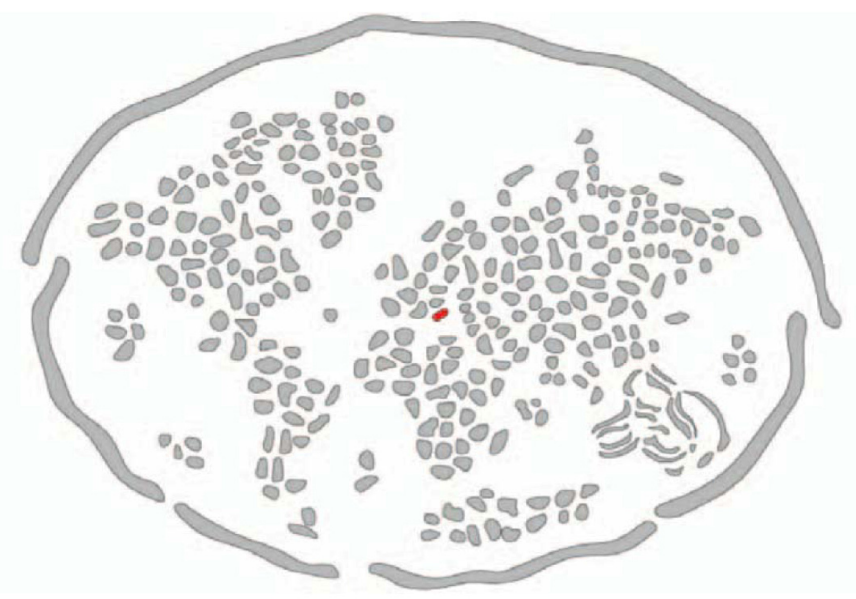

Figure 1: Masterplan "The World". 


\section{Middle Eastern archetypes}

Research on natural ventilation and passive cooling solutions has been conducted since antiquity in the Middle East. The archetypes described below relate to shares of the building envelope that are typical of Middle Eastern tradition and that perform the function of solar or ventilation control: elements combine and form a relationship between the architecture and the spaces that have to be acclimatized by using natural mechanisms [2]. One can witness construction systems that use direct natural ventilation, in which elements of air collectionextraction communicate with the environments that are to be cooled, as well as construction systems that use indirect ventilation, such as passive geothermic cooling with caption elements communicating via an underground pipe.

\subsection{Malqaf}

The malqaf is an example of wind chapter widespread in densely built cities, where the thermal comfort depends mainly on ventilation. In these circumstances, urban density significantly reduces air speed and a common window is inadequate for effective ventilation. The malqaf openings face north in order to capture the cooler air. The channel of communication between the malqaf and the interior has an outlet in every room. Iranian malqaf have a rectangular shape, are built in brickwork, and are high (between 8 to 15 meters). The caption opening is made with a colonnade opening facing prevailing winds. The channel conveys air especially to underground chambers and then makes it rise again.

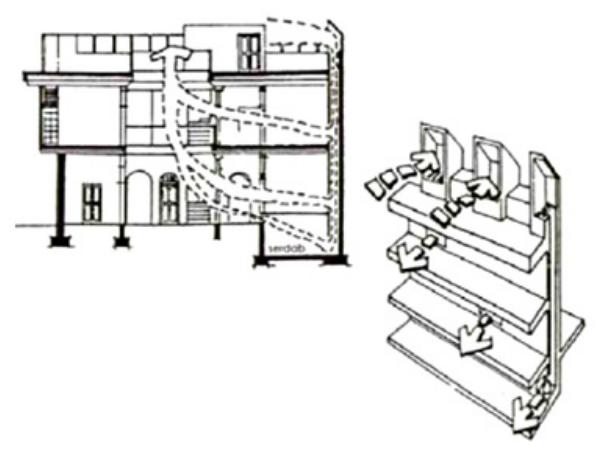

Figure 2: Malqaf.

\subsection{Bàdgir}

The bàdgir ("wind chapter" in Persian) is a system of multi-directional caption and extraction of wind, widespread in Iran and Persian Gulf areas. It consists of a tower with the summit opened on four sides, equipped with an internal vertical partition of bricks. The bàdgir functions to either capture the wind or to cool the flow as a result of a thermal mass that acts as a thermal flywheel mass: in the 
morning it is cooler than the air outside, which, in contact with the wall cools, becomes denser, and then drops down and enters the building. This process is accelerated in presence of wind. During the night the bàdgir returns the air absorbed during the day that, having moved into low areas of the building, has retained some of its freshness.

\subsection{Qà'a}

The $Q a^{\prime} a$ is a spatial element, whose name is of Turkish origin. The Q $\grave{a}^{\prime} a$ is an environment that is ventilated and cooled by a malqaf and a small lantern operating in synergy. Traditionally, it consists of three environments: one durQà'a, a central full-height space, with marble flooring and two iwanat, annex spaces with a floor level, covered with carpets, slightly raised in comparison to that of the $Q a^{\prime} a$, and where social life commences. At the cover of one of the two iwanat, usually the one facing north, a malqaf is placed. The system works by using the pressure difference between the systems involved: the malqaf, located in the windward side to the north and, therefore, under an excess of pressure, traps the air of the prevailing winds, that is cooler and faster than the inner one, channels it within the Iwan and, subsequently, into the dur-Qà'a. In that environment the air, meanwhile heated, tends to rise towards the small lantern placed on the ceiling, passing through the openings. Often, a fountain is placed in the centre of the room, in order to increase the humidity of the environment and lower the air temperature by evaporative cooling.

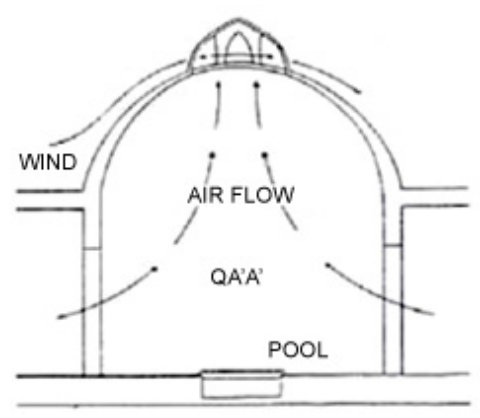

Figure 3: Qà'a.

\section{Passive design}

Passive buildings represent a more energy efficient type of construction. The design of energy efficient buildings is aimed at achieving perfect thermal insulation of the covering. These benefits are achieved with very careful planning, especially in terms of solar energy and high thermal insulation performance on the perimeter walls and glass surfaces, and through the use of ventilation energy recovery. The design philosophy adopted for these buildings is not easily standardized, because it changes depending on the site, local climate 
conditions in different seasons, natural resources available, socio-economic circumstances and habits in the area. Environmental, climatic, constructional, morphological, plant engineering, technological and behavioural aspects must all be in harmony, while improving the comfort of living.

\subsection{Site analysis}

The first step in designing a building that is energy-conscious and equipped with systems capable of capturing, storing and using the energy derived from external thermal solicitations, is a complete and structured analysis of the site and climate. This analysis entails accurately detecting the characteristics of the site area and collecting daily and monthly climate data. The main parameters that must be properly and dynamically analyzed are: air and soil temperature at different depths, windiness (wind intensity and direction), relative humidity, solar radiation, engineering design data, presence of trees or nearby areas intended for greenery, presence of natural or artificial watercourses, as well as other environmental factors. The design of passive buildings requires wellorganized implementation and use of appropriate computer software.

\subsubsection{Building orientation}

When designing buildings, it is essential to provide an optimum orientation in relation to solar radiation. Sun warmth and natural light are free renewable energy sources that can be used to increase housing welfare according to the season. As for the orientation of the building, a good solution is usually represented by a rectangular shape elongated along the east-west, with the living area of the building facing south.

\subsubsection{Presence of water}

The presence of water such as marine and ocean basins, lakes and large rivers, produces effects on the microclimate and, if large enough, even on the local climate, either in relation to thermal exchange or to air movements. The effects of the presence of water masses in the microclimate are related to different thermal properties of water and soil: water has a higher heat capacity. If radiated, it tends to warm up more slowly than soil, but releases the accumulated heat more slowly. This produces, essentially, two types of phenomena:

a. an attenuation of temperature range, either daily or seasonal, of the air above the water mass compared to that above the ground;

b. a temporal mismatch of the dynamics of thermal transfer between water and atmosphere.

To the thermal mitigation effect produced by masses of water, we must add the cooling effect produced by water evaporation during periods of overheating resulting from the contact with masses of warm air that lap its surface. The presence of water of a certain size produces typical local winds that originate from thermal gradients: coastal breezes, caused by the daily cyclic variation of 
atmospheric pressure determined by temperature differences and thus by air density. These breezes begin to rise during the day, when the earth warms up and the pressure difference increases. The differential pressure is reversed and decreases at night when ground temperature decreases more rapidly than that of water, causing a decrease in wind speed and a reversal in the flow.

\subsubsection{Winds}

Air flows through a building are generated by the differential pressure that is established between two or more openings, due to either wind or thermal gradient. This differential depends on the variation of characteristic air parameters: speed, direction and thermal gradient. Thus, before calculating air flows, one should define the parameters that characterize the variation of speed and pressure fields around a building.

\subsubsection{Vegetation}

The presence of plants can characterize the context of a building and its relative environmental quality, thus having a direct impact on the building itself. High trees and shrubs can play an important control function in the local microclimate, upon which thermal, luminous and acoustic comforts depend. This is because vegetation can play a barrier function to solar radiation, light, noise and prevailing winds. Furthermore, vegetation determines transpiration phenomena that influence air moisture and temperature. The solar control function performed by vegetable barriers is due primarily to their ability to intercept direct solar radiation, by absorbing it before it strikes the surfaces of the building covering, and by reducing the component reflected by the ground through the projection of shadows on it. At low latitudes, the sun remains high for much of the summer day, hence only a very broad and umbellifer coverage (the kind of maritime pine or palm) can have a real function for cooling soil, surfaces and air beneath the foliage. These tree species, by also having a rather high attack of the foliage, allow the flow of ground-level breezes that further contribute to the site cooling.

\section{Passive cooling}

Passive cooling of buildings includes all natural processes and techniques for the dissipation of heat through climatic resources in order to avoid the overheating

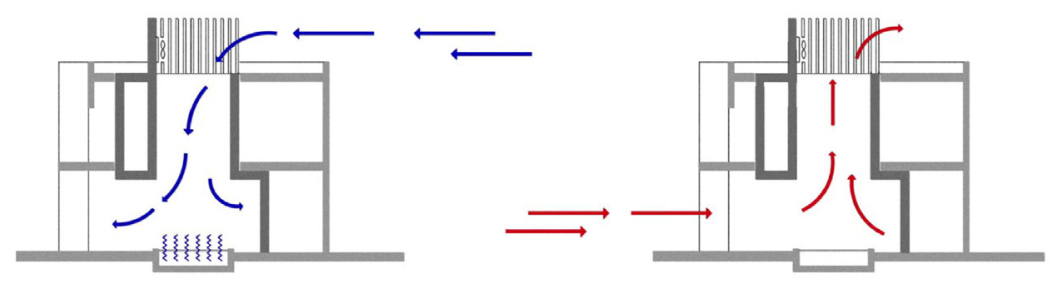

Figure 4: $\quad$ Input and output air. 
of a room. It strongly determines the comfort of occupants and may increase in efficiency if assisted and controlled by electronic and mechanical devices [2]. A more advanced cooling strategy leads to the elimination of heat in excess, through natural processes of heat transfer towards the upper atmosphere and the surrounding sky. Cooling takes over whereas high summer temperatures can cause environmental discomfort. It is possible to distinguish among the following main technical solutions:

- Soil Cooling: ground temperature is almost constant throughout the year and thus can be used to compensate heat loss through the building walls;

- Evaporative Cooling: in a warm-dry environment, adding moisture to air decreases its temperature of dry bulb, thereby improving comfort levels (this can be achieved by spraying water in a stream of air or by placing sheets of water or fountains in a courtyard);

- Induced Ventilation: the sun can be used to induce air movement (with a thermal chimney it is possible to activate natural ventilation and increase comfort levels).

\section{Control of solar radiation}

The need to illuminate interiors with natural lighting is quite clear. Aside from the purely energetic contribution, one should not underestimate the psychological and ecological benefits that are associated with the use of natural lighting. However, natural lighting exhibits two drawbacks that make it difficult to use: directionality and very high intensity [3]. Moreover, in tropical areas, where the environment produces clear sky conditions during the dry season and overcast conditions in the wet season, special consideration must be given to the adequacy of natural lighting. In such climates, the natural lighting of buildings depends on the need of excluding or limiting direct exposure to sun light, which represents a thermal gain.

\subsection{Sun-shading system}

Shields have an important role in solar control since it is very difficult to reach a sufficient level of solar protection using only high performance covering technologies. Shields, if planned in relation to the seasonal path of the sun, can offer effective summer protection, helping to ensure comfort conditions all year around. There are numerous factors that must be considered when designing a screen. Among the major elements we can list: the latitude of the site, the orientation of the surface to be protected, and the location where the shield itself is planned to be placed. Types of shielding are varied and diverse and can represent significant elements of architectural language. The positioning of a screen is a major factor for thermal control: if placed outside of the transparent surface, its effect is greater than inside, as it intercepts solar radiation before it enters in the building, allowing outside air to disperse absorbed heat. If placed inside of the transparent item, radiation, although it does not directly hit the surfaces of the internal environment, is absorbed by the individual elements of 
the screen, which by warming, transfers the heat inside, by radiation and convection, thus adversely affecting the energy balance of the summer. The effectiveness of the screen varies, therefore, considerably. In particular, we must consider:

a. the proportion of intercepted solar radiation in comparison to the incident one, which depends on the geometry and management of the screen;

b. the remission of infrared of heat absorbed by the elements that make up the screen, depending on the location, the equipment and its characteristics.

\subsubsection{Psychophysical wellness}

The presence of shielding has direct effects on the perception of the internal environment by the occupants. In the selection and design of the shielding system, as mentioned above, it is necessary to consider the different needs associated with the presence of individuals within rooms and the characteristics of the activities are conducted within those rooms. In particular, we must consider the requirements associated with the following areas:

- $\quad$ Thermal Scope

- containment of energy consumption for cooling and heating (solar control in summer and winter);

- control of air temperature within comfort levels;

- limiting people's direct contact with solar radiation;

- Lighting Scope

- control of the level of natural lighting;

- $\quad$ test of the distribution of lighting levels;

- limitation of glare effects;

- Perceptual Scope

- internal-external vision by users.

The designer must choose the type of screen by weighing, from case to case, these needs and trying to minimize conflicts, dictated by the opposite character of some of them. Each technological solution, in fact, will be the right compromise in relation to the intended use of the rooms.

\section{Solar energy}

Solar radiation can be used in buildings and homes to directly produce electricity, hot water for sanitary use, and/or under floor heating systems. The energy that can be recovered through solar collectors is influenced by a number of factors, such as the overall availability of solar energy and the performance in transforming this energy (influenced by the type and placement of collectors). The efficient use of this form of energy affects the overall design of buildings that must be designed from the onset to adequately capture and store the heat from direct sunlight. The photovoltaic system is the only proven technology for the production of electricity from solar sources. In recent years, the production of such modules has experienced growth rates exceeding 30\% per annum. 


\subsection{Solar collectors}

Buildings that have a sunny area (flat roof, sloping roof, garden, etc.) can be equipped with solar systems. The yield of a solar heating system depends on several factors: the local weather conditions, area and type of system installed, heat load, etc. In order to achieve maximum efficiency, these systems are placed in the best possible position in an easily accessible area, non-critical in the anchorages and able to make the most of solar radiation. The collectors should preferably be directed southwards, with a tolerance of deviation to the East or West of $30^{\circ}$. Solar thermal systems can be based on natural circulation or on forced circulation. Natural circulation solar systems consist of collectors and a tank mounted on the same chassis. This is a closed loop system that works on indirect exchange and in which the circuit of the heat transfer fluid is always separated from the water going directly to users. In such systems, mechanical or electrical equipments are not necessary for operation: the sun is the only source of energy for which they are equipped. The thrust of the thermo-conveyer fluid is given by the difference in density between the column of the hotter fluid that tends to rise and the colder column coming out of the tank that tends to fall. The fluid, through a cavity of the shell, releases heat to the water contained therein that flows into the circuit of users. Forced circulation systems are, by contrast, more complex and therefore require more maintenance. For systems with natural circulation, a lower initial investment and minimal maintenance are required and, therefore, are a recommended solution for small users. The installation of forced circulation is rather realistic for every demand, but requires careful monitoring of its performance and is therefore a recommended solution for medium and large facilities with a technical staff for routine maintenance. The main use of solar collectors is inherent in the heating of water for sanitation purposes, but their use can be extended to great advantage in all situations that require heat at low temperature. For example, their use is particularly suitable for heating swimming pools.

\subsection{Photovoltaic plants}

$\mathrm{PV}$ is a renewable source that can help cover the electrical needs by reducing pollutant emissions. The photovoltaic effect can convert sunlight into electricity through the use of photovoltaic cells composed of a semiconductor properly treated (usually the pure silicon). There are two types of modules on the market: crystalline silicon and thin film. Crystalline silicon modules are formed by assembling several cells together. They can be divided into modules with monocrystalline silicon cells or polycrystalline silicon cells. The mounting frame which supports the modules must guarantee a certain resistance to wind and temperature changes, and ensure a lifetime of more than 20 years. The assembly of modules and connections should be as simple as possible and allow the replacement of a module or more modules, without having to disassemble the entire plant. 


\section{Insulating PCM}

Often called PCM, "Phase changing materials," are insulating materials which are intelligent accumulators of heat, exploiting the physical phenomenon of phase transition for absorbing latent heat energy flows and storing a large amount of energy, keeping constant its temperature, and returning the heat outside during a subsequent lowering of temperature. In fact, PCM are solid capsules at room temperature, but when the temperature exceeds a certain threshold, they melt accumulating heat, which is subtracted from the environment. The PCM are a product of NASA research, and only recently have been applied for energy saving in the construction sector. The materials that contain PCM may differ - plasterboard, wood, plaster, plexiglass, and can be applied in various system solutions, such as heating, cooling, solar collectors and heat exchangers. They can also be contained in a layer of plaster, thus allowing the maintenance of pleasant temperatures inside buildings. These wax microcapsules are protected by plastic wrap, so as not to spill out of the plaster during the winding phase.

\subsection{Fibreglass insulation - the Nanogel}

Nanogel [4] systems are also very innovative and are able to achieve high levels of isolation combined with characteristics of lighting diffusion. They were introduced by the United States in Europe in early 2000, and are used to make public and sports buildings, since they have particular characteristics of lighting diffusion, avoiding shadows and the use of externally applied shielding. Special sandwich panels have an internal aluminium structure or a fibreglass grid with the cavity completely filled with Nanogel. This is an insulator composed of silicon micro-grains and nanoscale-pores that make it similar to a sponge composed of $95 \%$ air, with a thermal transmittance of $0.28 \mathrm{~W} / \mathrm{mqK}$ for a thickness of $70 \mathrm{~mm}$. This value of transmittance is much greater than that of a traditional glazing system, it is in fact similar to an opaque wall, but is much less thick. With this technology it is possible to manufacture large surfaces that are permeable to natural lighting and are capable of ensuring high levels of insulation and energy saving.

\section{ECOTECT v5: software for sustainable design}

ECOTECT v5 is the most comprehensive and innovative program on the market today for the verification of buildings. ECOTECT [5] allows application of the principles of environmental design starting from the conceptual and ideational phase of the project. The program has an interface for 3D modelling, integrated with functions for control of acoustics, thermal insulation, lighting, solar energy contribution and costs. What really distinguishes the program is that it can serve as a support throughout the entire project phase, from testing early theoretical elaborations to last stage verifications. It is possible to get useful information before the shape of the building has been fully developed. Detailed analysis on 
the climate can be used to optimize available resources of light, wind, and sun, thanks to the interpretation of models sketched, which are easily analyzed and compared. Verification is done in detail at any stage of design. The most significant feature of ECOTECT is the interactive approach to the analysis of data. It is possible to add a window and immediately see the effects on thermal insulation, compared to the benefits in terms of natural lighting, solar radiation accidents, and the overall cost of the building.

\subsection{Hourly solar exposure}

The graph shows a single image in the global hourly radiation available on the selected surface, its share in the shadows, the value of the incident and reflected radiation of objects in the model that are set as solar reflectors. The global radiation is calculated according to the geographical location of the project, which refers to the climate data file previously loaded into the model. The direct radiation is the proportion of solar radiation that affects the selected surface, while the scattered radiation is the part of solar radiation emitted by the vault of heaven.

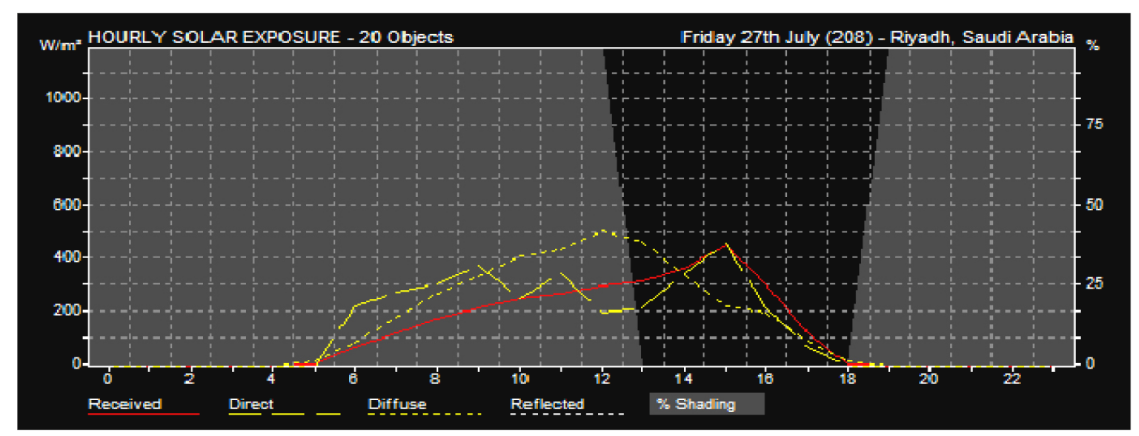

Figure 5: Hourly solar exposure graph.

\subsection{Received solar radiation}

For each month of the year, the average daily solar radiation that strikes a specific area of the model is determined. The total solar radiation for each month of the year is calculated from climate data file reference and then divided by the respective number of days. The graph shows the resulting hourly average values that might be expected on any day of the month: the warmth of each cell represents the intensity of solar radiation striking the selected surface in a precise time of an average day of a given month of the year.

\subsection{Hourly temperatures}

This graph shows the time evolution of temperature within each zone on the date selected. Dashed and dotted lines represent the data of the climate of that day, as explained by the legend directly below the graph, while the solid line shows the 
trend of the temperatures inside the rooms. The temperature of the chosen area is highlighted in double thickness. Gradients red and blue in the graph indicate when the temperatures in the area are below or above the level of comfort, respectively.

\section{Conclusion}

The combination of the described technologies following the global approach of the sustainable design, allowed one to reduce the global energy consumption of the SPA of about $30-35 \%$ with a consistent reduction of $\mathrm{CO}_{2}$ emissions and of land resource consumption. It is important to underline how an extreme climate, such as the one in Dubai, permits to obtain the maximum performances from almost all the described technological systems. The substantial reductions of the realizing, the management and the environmental costs of the buildings are demonstrated by the first results of the compared LCA analyses actually performed in CITERA laboratories.

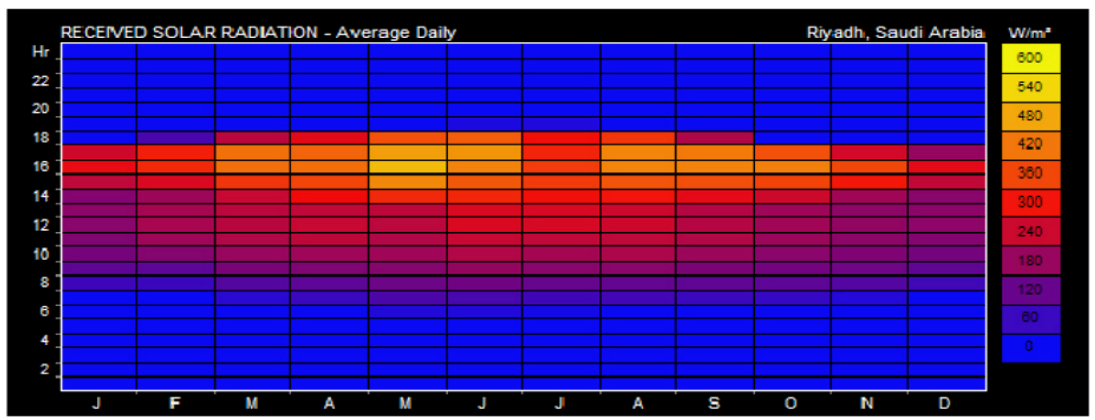

Figure 6: Received solar radiation graph.

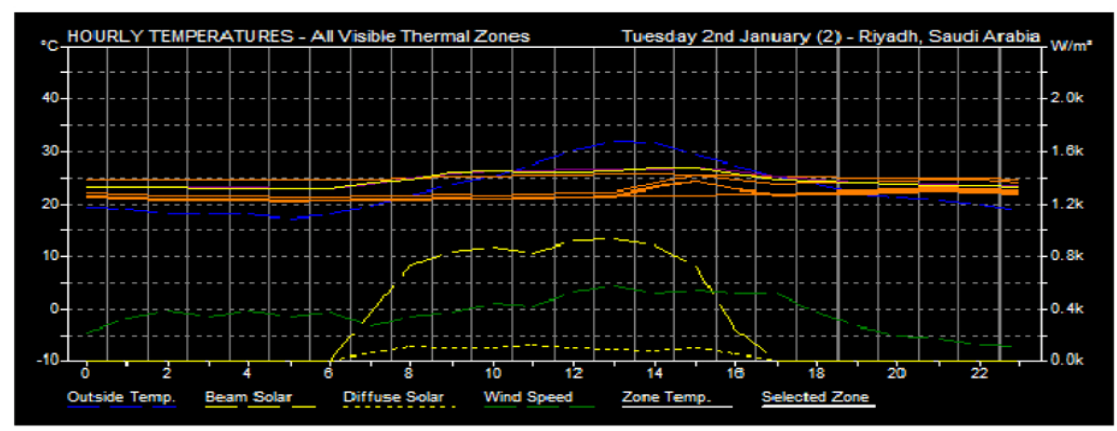

Figure 7: Hourly temperatures graph. 


\section{References}

[1] Assante E., Dubai, Las Vegas in the Persian Gulf, La Repubblica: Roma, 11 Agosto 2008, intro.

[2] Pilotti L., Le torri iraniane: un mirabile esempio dell'arte del costruire, Urban Review n44, 10/22/2007, 2.

[3] Grosso M., Passive cooling in temperate zone, Maggioli 2008, 4.

[3] Hopkinson, Control of solar radiation, 1966, 5.

[4] Spagnolo M., The future of ultra-efficient materials www.rinnovabili.it, 18 January 2008, 7(1).

[5] Urso M., Integrated simulation solutions bioclimatic- ECOTECT software sustainable design construction. ISES Italia, 2007, 8. 